\title{
A Personal Perspective on the Unconventional Superconductivity of $\mathrm{Sr}_{2} \mathrm{RuO}_{4}$
}

\author{
A. P. Mackenzie ${ }^{1,2}$ (D) \\ Received: 1 July 2019 / Accepted: 13 September 2019 / Published online: 7 November 2019 \\ (C) The Author(s) 2019
}

\begin{abstract}
I give a personal account of the unfolding story of the unconventional superconductivity of $\mathrm{Sr}_{2} \mathrm{RuO}_{4}$. This is a subject of topical importance in light of recent measurements that suggest that its order parameter may be even parity, contrary to the picture that had built up over the course of over two decades of research. With an eye on the past, I stress the generous encouragement that I received from Ted Geballe in the early years of my $\mathrm{Sr}_{2} \mathrm{RuO}_{4}$ research. Looking to the future, I give my opinion about why the $\mathrm{Sr}_{2} \mathrm{RuO}_{4}$ problem is of major significance to the development of the field of unconventional superconductivity, whatever order parameter symmetry is finally established to be the correct one.
\end{abstract}

Keywords $\mathrm{Sr} 2 \mathrm{RuO} 4 \cdot$ Unconventional superconductivity

I should start by saying that it is a pleasure and an honour to contribute to this most special of volumes celebrating the career of a man for whom I have the utmost personal respect. For me, Ted Geballe embodies all that is good in the world. His brilliant scientific insight is accompanied by immense and completely spontaneous generosity and kindness, whether in the professional or personal arena.

As my contribution, I think that it is appropriate that I write a short perspective on the unconventional superconductivity of $\mathrm{Sr}_{2} \mathrm{RuO}_{4}$. There is no shortage of review articles on $\mathrm{Sr}_{2} \mathrm{RuO}_{4}$ [1-5]. I certainly do not intend to repeat the content of those here, but for several reasons that I hope will become clear, I think that the time is right for me to write a short personal article. The first reason for this choice is that, like Ted, $\mathrm{Sr}_{2} \mathrm{RuO}_{4}$ superconductivity is celebrating a milestone birthday. It is a trivial one compared to his, but 2019 marks a quarter of a century since Yoshi Maeno and colleagues' landmark discovery in Hiroshima [6] of superconductivity in

A. P. Mackenzie

andy.mackenzie@cpfs.mpg.de

1 Max Planck Institute for Chemical Physics of Solids, Dresden, Germany

2 Scottish Universities Physics Alliance, School of Physics and Astronomy, University of St Andrews, St Andrews, UK high-quality single crystals grown a few years earlier by Frank Lichtenberg in Georg Bednorz's group at IBM Zürich.

I had met Yoshi at a conference in 1993, and he knew from those discussions that I had an interest in low temperature measurements. He therefore sent me a preprint of his 1994 paper (in those days still a paper item brought to me by a colleague who met him in Japan) and I was fortunate to establish a collaboration with him to study the normal state properties of $\mathrm{Sr}_{2} \mathrm{RuO}_{4}$ even before his paper appeared in print. Those were truly exciting times, with the de Haas-van Alphen (dHvA) effect oscillations that had stubbornly eluded observation in the cuprates rising high above the background noise in $\mathrm{Sr}_{2} \mathrm{RuO}_{4}$. My own work, carried out in Cambridge in close collaboration with Stephen Julian [7] and, later, Christoph Bergemann [8, 9], was mainly on dHvA and other normal state properties, but I did have the chance to work on one aspect of the superconductivity.

On a visit to Hiroshima, one of Yoshi's graduate students, Koji Yoshida, casually mentioned to me that they did not always manage to obtain superconductivity in the crystals that they grew. By that time, the field was abuzz discussing the brilliant suggestion by Maurice Rice and Manfred Sigrist that the superconductivity of $\mathrm{Sr}_{2} \mathrm{RuO}_{4}$, though low- $T_{\mathrm{c}}$ with transitions at approximately $1 \mathrm{~K}$, might be unconventional in nature. Specifically, based on analysis of the normal state data, they proposed that $\mathrm{Sr}_{2} \mathrm{RuO}_{4}$ might be a spin triplet superconductor, in analogy with the pairing in superfluid ${ }^{3} \mathrm{He}[10]$. I knew little-very little indeed!-about triplet superconductivity 
and vector order parameters, but I had picked up from discussions with my PhD supervisor Gil Lonzarich that if that were really the case, one expected the superconducting transition temperature to be extremely sensitive to non-magnetic disorder. My ears therefore pricked up when Koji told me about the sample-sample variations, and I returned to Cambridge with samples from across the range of growth batches. In one of the great coincidences that often shape scientific progress, I had exactly the right expertise to investigate a problem like this, because in the absence of cuprate $\mathrm{dHvA}$ oscillations, my $\mathrm{PhD}$ had ended up as a highly technical one on using electron probe microanalysis to study the chemical composition of difficult oxides. The Cambridge Earth Sciences department had a stateof-the-art instrument for these purposes, which featured Bragg spectrometer x-ray detectors that enabled excellent signal-tonoise detection of trace elements. It was in the charge of one of the founders of that field, Stephen Reed, from whom I learned a lesson that I still pay heed to today - if at all possible, do not place a technician between a sophisticated instrument and its users. Instead, give advice and tuition, but allow them the freedom to really use it themselves. Because of Stephen's enlightened attitude, I had been given the time to learn how to programme it to perform long, bespoke data taking runs. A quick bit of coding and about 2 weeks of data acquisition time later, we had good evidence that although the crystals were all nominally being prepared in the same way, they had significant variations in the levels of non-magnetic $\mathrm{Al}$ and $\mathrm{Si}$ impurities. This fired us up to perform measurements correlating the resistive mean free path with the superconducting transition temperature, on a dilution refrigerator set up for other reasons by Kurt Haselwimmer in Cambridge. The result, shown in Fig. $1 \mathrm{a}$, provided what I believe is conclusive evidence that $\mathrm{Sr}_{2} \mathrm{RuO}_{4}$ has an unconventional order parameter. It is important to mention that this does not prove that $\mathrm{Sr}_{2} \mathrm{RuO}_{4}$ is a triplet superconductor, just that it is unconventional. Any odd parity spin triplet state can be destroyed by non-magnetic scattering, because randomizing the phase of the order parameter averages it to zero, but the same logic applies to even parity $d$ - (or $g-\ldots$ ) order parameters as well.

I had enjoyed that experiment and was vaguely aware of its potential significance, but I was not rushing to write it up.
My reluctance was partly due to pressure of work on the normal state properties and partly because I was concerned that, with only seven data points for superconducting samples, its apparent conclusions might simply be wrong! At that stage of the project I had the privilege of discussing it with Ted, and his enthusiasm and encouragement were absolutely key to driving me on to writing the paper [11]. As a young scientist, I learned so much from those early discussions with Ted, and I have tried hard to remember them as I grow older (though still a spring chicken in his terms!). Not coming from the US community, and being badly ignorant about scientific history, I really had not known his incredible pedigree until talking with him. He liked the work because, to him, it was a good example of real life materials physics. We all know that he is extremely strong in terms of chemical intuition, but he was so knowledgeable about superconductivity theory as well, and he instantly recognized the significance of the data seeming to follow the Abrikosov-Gorko's pair breaking function. The thing that really came through to me from the conversations was his ability to inspire and the simple way he achieved that. He is always tough on scientific matters, but he is also unfailingly kind and constructive and has the precious ability to make young people feel good about themselves and the work they are doing. I try to act in a similar way when I can, and often cross-refer to him as my example.

Yoshi Maeno is a consummate materials physicist (also influenced by Ted of course!) and he reacted to our findings by leading his group's refinement of the sample preparation techniques to the level where it became possible to grow crystals with $T_{\mathrm{c}} \sim 1.5 \mathrm{~K}$ as predicted by extrapolation of the pair breaking function to the high purity limit [15]. That refinement continues to this day [16], and means that single crystals of $\mathrm{Sr}_{2} \mathrm{RuO}_{4}$ with mean free paths of microns are available for the world-wide collaborative research that Yoshi has stimulated by his wonderfully unselfish attitude to sharing the crystals with other groups. The refinement in crystal quality in turn made it possible to deliberately disorder pristine crystals with controlled levels of impurities introduced during the growth process, and hence for a number of groups to check the validity of the data we had published [12-14]. To my relief, our
Fig. 1 a Original data from our group on the purity dependence of $T_{c}$ in $\mathrm{Sr}_{2} \mathrm{RuO}_{4}$ [11]. b The results of some of the follow-up confirmatory work by other groups [12-14].
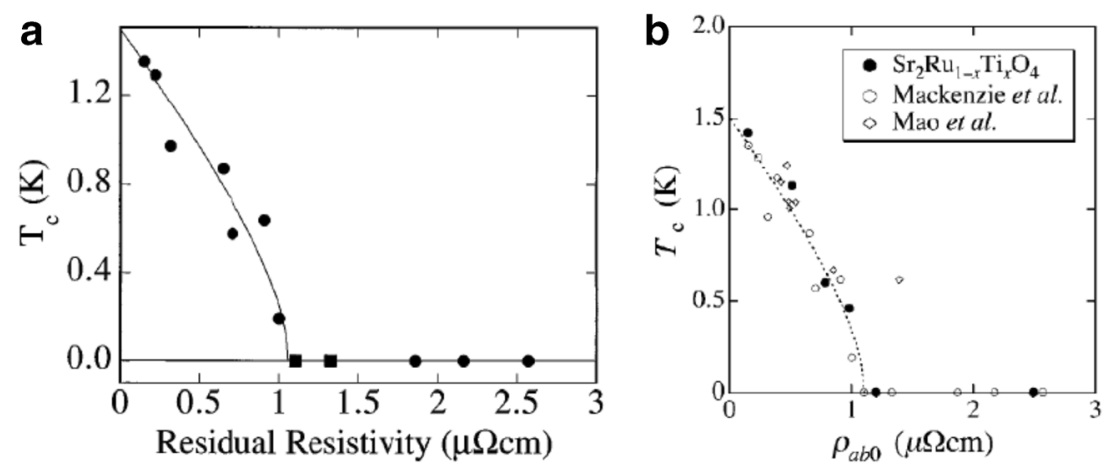
work stood up to that scrutiny, as evidenced by the data shown in Fig. 1b.

'Living' an experiment the way we lived the impurity one on $\mathrm{Sr}_{2} \mathrm{RuO}_{4}$ is a good way of maximizing what one can learn from it, and it helped me form the opinion that dirty samples are hiding legions of unconventional superconductors that we would be able to discover in the clean limit. The disordersensitivity of other unconventional superconductivity has been noted or studied before and since our work, but to the best of my knowledge $\mathrm{Sr}_{2} \mathrm{RuO}_{4}$ still holds the record as the superconductor with the most stringent and proven mean-freepath criterion $\left(>\sim 10^{3} \AA\right)$ for the existence of the superconductivity. That that should be the case comes as something of a surprise, and I look forward to the record being broken soon!

Our experiment showing that $\mathrm{Sr}_{2} \mathrm{RuO}_{4}$ must have an unconventional order parameter was followed very quickly by two highly influential pieces of work that have largely defined the field until the last year. In the first, Luke and collaborators presented muon spin rotation evidence that a spontaneous magnetic field was generated in the superconducting state of $\mathrm{Sr}_{2} \mathrm{RuO}_{4}$, implying an order parameter in which time reversal symmetry is broken [17]. Shortly afterwards, Ishida and collaborators reported that the NMR Knight shift remained unchanged as the sample is cooled through $T_{\mathrm{c}}[18]$. This strongly implied that the spin susceptibility was the same in the superconducting and normal states, something that is impossible in the presence of spin singlet pairing for which the formation of non-magnetic singlets suppresses the spin susceptibility. Both these observations were consistent with one of the triplet order parameters suggested in the classic prediction of Rice and Sigrist, and both were subsequently confirmed in other experiments $[19,20]$. Combined with evidence from tunnelling experiments for two-component order parameters [21] with odd parity [22], this all seemed to add up to compelling evidence for a real 'feel-good' situation-a superconductor whose order parameter was a two-dimensional version of that of the A phase of superfluid ${ }^{3} \mathrm{He}$. This excitement of this possibility gained further momentum with the publication of a second beautiful theoretical idea, namely that such a superconductor can host topologically protected Majorana zero modes in its vortex cores [23]. It was clear that the superconducting gap contained nodes or very deep gap minima which would severely restrict any practical use of the Majorana modes [24], but as long as the nodes were accidental they did not rule out a topological state.

When a beautiful hypothesis exists in some physical situation, it is always tempting to use it as the reference interpretation for subsequent work. This certainly happened in the case of $\mathrm{Sr}_{2} \mathrm{RuO}_{4}$, for which many experiments over the next decade were shown to give results consistent with the prevailing received wisdom, without, however, providing clear proof in favour of it. Balancing these results were another set of experiments that gave reasons for doubt. For example, neither the critical field splitting expected near $T_{\mathrm{c}}$ nor the internal fields or edge currents predicted naively expected for a time reversal symmetry breaking superconductor were observed $[25,26]$. Some ingenious theoretical work $[27,28]$ showed that the edge currents need not be a firm expectation in a multi-band superconductor like $\mathrm{Sr}_{2} \mathrm{RuO}_{4}$, so it was difficult to judge whether these counter-experiments were fatal to the received wisdom - was the cup half full or half empty?

Probably my biggest personal concern about the situation was another discrepancy concerning the anisotropy of the critical magnetic fields. In a strongly two-dimensional superconductor, the critical field anisotropy should be calculable from knowledge of the anisotropy of the Fermi surface. I was pretty confident that our de Haas-van Alphen experiments had established the Fermi surface anisotropy, and it suggested that the critical field anisotropy should be very large - at least a factor of 100 . What was measured was approximately a factor of 40 [1]. One would not normally worry about a factor of 2.5 numerical discrepancy, but it was also established that there is a first-order phase transition out of the superconducting state at high magnetic fields $[29,30]$. Taken together, these suggested that the critical fields were behaving as if they were limited by the Zeeman energy of breaking Cooper pairs. This would be fine in a standard superconductor but was in direct contradiction to the Knight shift results which suggested that there should be no Zeeman energy gain from breaking Cooper pairs.

Throughout the decade or more during which the abovedescribed situation was developing, my own group was doing little or no work on $\mathrm{Sr}_{2} \mathrm{RuO}_{4}$ superconductivity, and I played the role of an interested observer. In late 2012, however, we started to work in the field again, but from a different angle. Following a workshop discussion with Jim Sauls, my then post-doc Clifford Hicks proposed a new way of applying uniaxial pressure that would in principle enable a number of desirable things, namely application of both compressive and tensile pressure, pressure actuation at low temperatures based on piezo-electric stacks, and, in ultimately favourable circumstances, the generation of large strains of order $1 \%$ or more. It seemed to me that so many things could go wrong that the apparatus was pretty unlikely to work, but I agreed with Clifford that we should put some time and resource into it, in the hope that my pessimism would be unfounded. Happily it was [31].

The original motivation for the uniaxial pressure project that emerged from the conversation with Jim Sauls was to search for one of the expectations for a time reversal symmetry breaking order parameter, namely a cusp in $T_{\mathrm{c}}$ at zero uniaxial pressure. As is so often the case with experiments designed to search for some specific phenomenon, we did not observe it, either in the original experiment [32] or a more sophisticated follow-up in collaboration with the group of Ted's colleague Kathryn Moler at Stanford [33]. However, 
the experiment reaped rewards that we had not thought of when we conceived it. It was possible to increase $T_{\mathrm{c}}$, not by a few $\mathrm{mK}$ but by a large factor of 2.4 , up to approximately $3.5 \mathrm{~K}$ [34]. We have subsequently performed follow-up measurements showing that this increase of $T_{\mathrm{c}}$ is due to pressure tuning through a Lifshitz transition [35], but even without this microscopic understanding, the experiment enabled a reexamination of the critical field limiting. What had been a worrying but numerically fairly small discrepancy in the asgrown material would be a major contradiction in $T_{\mathrm{c}}=3.5 \mathrm{~K}$ material if it had a spin triplet order parameter [34].

Disagreement between experiment and theory is both common and healthy in correlated electron physics for which developing appropriate many-body theory is one of the big challenges for twenty-first century physics. Strong apparent disagreement between the results of two complementary experiments is a different level of discrepancy and merits careful investigation. Working with Thomas Scaffidi, Clifford and Yoshi Maeno, we stressed this and other puzzles in a 2017 review article that emphasized the importance of re-examining the received wisdom about the order parameter of $\mathrm{Sr}_{2} \mathrm{RuO}_{4}$ [5]. This challenge was taken up by Stuart Brown at UCLA, whose group set out to study the Knight shift of $\mathrm{Sr}_{2} \mathrm{RuO}_{4}$ under uniaxial pressure, in collaboration with our group in Dresden. The striking results obtained in that project are my main motivation for writing this short perspective. Stuart and colleagues began by demonstrating that, in the pressurized $T_{\mathrm{c}}=3.5 \mathrm{~K}$ material, the Knight shift does not remain constant below $T_{\mathrm{c}}$, but shows a significant drop that is qualitatively consistent with expectation for singlet rather than triplet pairing. This observation naturally raised the question of what was happening at ambient pressure. In a series of careful experiments, they showed that the famous previous result from Ishida's group was due to transient heating and that in fact the
Knight shift changes strongly below $T_{\mathrm{c}}$ at all pressures [36]. At a stroke, the discrepancy between the NMR and critical field limiting experiments has been removed!

One of the real pleasures of working on $\mathrm{Sr}_{2} \mathrm{RuO}_{4}$ has always been the interaction with the world-wide community of colleagues who find the material as fascinating as I do. We do not always agree on the science or interpretation, but we work in an atmosphere of constructive discussion and mutual respect. In other fields that I have observed or worked in, a result like Stuart's that overturns a received wisdom can be the prelude to years of unconstructive dispute that obstructs scientific advance. For me, Ted is a guardian of the standards of scientific behaviour, so I am sure that he will approve of what happened in this case. We made sure, before posting our results on the arXiv, that we had described Stuart's experiments in detail to Kenji Ishida. He freely admitted that they came as an unwelcome shock to him, but he reacted in the most admirable way. He went back into the laboratory personally, rechecked his experiments in light of Stuart's findings, and contacted us within weeks to confirm his agreement with the new results. At time of writing he has placed a detailed paper on the arXiv describing what he has done [37].

In my opinion, these very recent developments leave us at a key stage in the quest to understand the unconventional superconductivity of $\mathrm{Sr}_{2} \mathrm{RuO}_{4}$. They make it likely that $\mathrm{Sr}_{2} \mathrm{RuO}_{4}$ is not an odd parity, spin triplet superconductor but an even parity, spin singlet one. It is interesting to observe the reaction of colleagues to this new development. At one level, it comes as a disappointment; a solid-state superconducting analogue to the A phase of superfluid ${ }^{3} \mathrm{He}$ is such a nice idea! Understandably, people who have worked intensively on that idea, and/or on experiments whose results aligned well with it, are reluctant to change their world view immediately. That is entirely appropriate, and a re-examination of some of the other
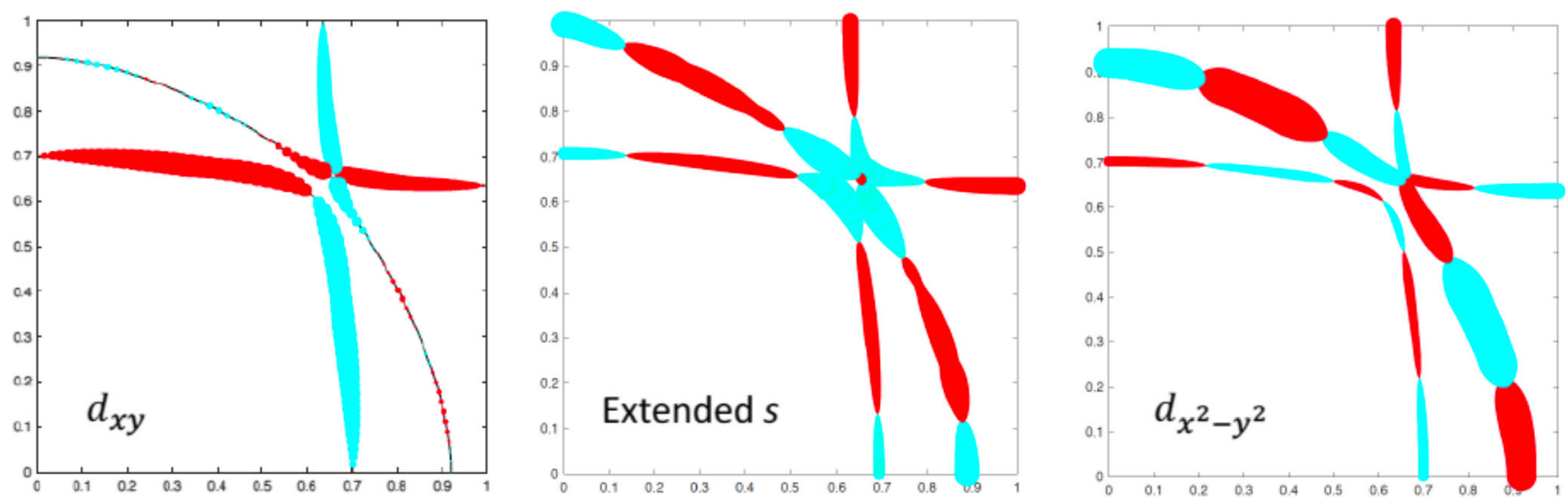

Fig. 2 Illustrative calculation of possible gap structures in $\mathrm{Sr}_{2} \mathrm{RuO}_{4}$ by Thomas Scaffidi, based on the weak-coupling model of ref. [38]. In each case, one quadrant of the $2 \mathrm{D}$ square Brillouin zone is shown, with sign reversals of the order parameter denoted by changes from red to blue.
While not identical, the nodal structures of the three candidate even parity states some striking similarities that run counter to the intuition that one would have based on single-band thinking. 
existing results should be a high priority for the future. However, it is important that this is all done with cool heads and open minds. The extreme viewpoints of either clinging on to the triplet interpretation or throwing it out of the window without trying to revisit the outstanding mysteries would be equally unhelpful as the field moves forward. It seems to me that the two biggest issues will be establishing whether time reversal symmetry is really broken at $T_{\mathrm{c}}$, and increasing efforts to perform measurements sensitive to parity.

Arguably, the biggest issue of all moving forward is whether the effort of doing so is merited. A number of people question whether $\mathrm{Sr}_{2} \mathrm{RuO}_{4}$ warrants further study at all if it does not turn out to be the ${ }^{3} \mathrm{He}$ analogue of our dreams. This is a perfectly valid issue to raise, but it is one on which I hold a very strong opinion: actually, it is vital that this work be done. For several reasons, it goes to the very heart of how unconventional superconductivity condenses from correlated electron metallic states. $\mathrm{Sr}_{2} \mathrm{RuO}_{4}$ is the cleanest of all known unconventional superconductors and has one of the simplest and best understood normal states. It is an ideal test-bed for theoretical ideas and for establishing the validity of information coming from the key experiments that are widely applied to unconventional superconductors. If these experiments give contradictory results in a material like $\mathrm{Sr}_{2} \mathrm{RuO}_{4}$, how can we just assume that they are reliable when applied to other materials? Although it is sometimes convenient to assume that reliability and use it to assign order parameter classifications based on sparse information, that approach will not lead to progress in the long run. The second reason that $\mathrm{Sr}_{2} \mathrm{RuO}_{4}$ should remain as an archetype for the field is as a guide to the development of theory. Its low $T_{\mathrm{c}}$ makes the use of weakcoupling theory more justifiable on $\mathrm{Sr}_{2} \mathrm{RuO}_{4}$ than on most other unconventional superconductors, and the application of that theory within a two-dimensional approximation has already been very revealing. The free energy differences between odd- and even-parity superconducting states is small, almost certainly below the true accuracy (including the validity of assumptions made) of the calculations. In other words, the $\mathrm{Sr}_{2} \mathrm{RuO}_{4}$ problem is a hard one, and establishing the true experimental picture beyond doubt is therefore a vital guide to the development of theories of the mechanism of unconventional superconductivity as a whole.

To frame that quest I will make unusual move of closing this perspective with a second figure, courtesy of my colleague Thomas Scaffidi. Based on the weak-coupling approach of ref. [38] which includes spin-orbit coupling, it shows typical calculated gap functions for three distinct even parity order parameters, $d_{x y}$ extended $s$ and $d_{x^{2}-y^{2}}$. In simple single-band thinking, these order parameters would have completely different nodal structures, but in three-band $\mathrm{Sr}_{2} \mathrm{RuO}_{4}$ the distinction is much weaker. Symmetry-imposed nodes are of course present, but each state also has a rich structure of accidental nodes. The overall message to be taken from inspection of Fig. 2 is that, in a model unconventional superconductor in the weak-coupling limit, the nodal structure is determined not by symmetry alone but by its combination with a complicated spin fluctuation spectrum, with the latter being arguably more influential on the overall nodal structure than the former. Even if the particular theory that led to Fig. 2 is not the perfect theory of $\mathrm{Sr}_{2} \mathrm{RuO}_{4}$, this is a sobering observation, because it warns us that the relationship between standard physical observables and order parameter symmetries may not be nearly as simple as we usually assume, either in $\mathrm{Sr}_{2} \mathrm{RuO}_{4}$ or many other unconventional superconductors. For this and other reasons, unravelling the $\mathrm{Sr}_{2} \mathrm{RuO}_{4}$ problem is, in my opinion, more important than ever. However, I am optimistic. The new NMR results have resulted in a paradigm shift in the field, but a welcome one that should be viewed as opening previously closed paths to an overall understanding.

Funding information Open access funding provided by Max Planck Society.

Open Access This article is distributed under the terms of the Creative Commons Attribution 4.0 International License (http:// creativecommons.org/licenses/by/4.0/), which permits unrestricted use, distribution, and reproduction in any medium, provided you give appropriate credit to the original author(s) and the source, provide a link to the Creative Commons license, and indicate if changes were made.

\section{References}

1. Mackenzie, A.P., Maeno, Y.: The superconductivity of $\mathrm{Sr}_{2} \mathrm{RuO}_{4}$ and the physics of spin-triplet pairing. Rev. Mod. Phys. 75, 657$712(2003)$

2. Maeno, Y., Kittaka, S., Nomura, T., Yonezawa, S., Ishida, K.: Evaluation of spin-triplet superconductivity in $\mathrm{Sr}_{2} \mathrm{RuO}_{4}$. J. Phys. Soc. Jpn. 81, 11009 (2012). https://doi.org/10.1143/JPSJ.81. 011009

3. Kallin, C.: Chiral p-wave order in $\mathrm{Sr}_{2} \mathrm{RuO}_{4}$. Rep. Prog. Phys. 75, 42501 (2012). https://doi.org/10.1088/0034-4885/75/4/042501

4. Liu, Y., Mao, Z.-Q.: Unconventional superconductivity in $\mathrm{Sr}_{2} \mathrm{RuO}_{4}$. Phys. C-Supercond. Its Appl. 514, 339-353 (2015). https://doi.org/10.1016/j.physc.2015.02.039

5. Mackenzie, A.P., Scaffidi, T., Hicks, C.W., Maeno, Y.: Even odder after twenty-three years: the superconducting order parameter puzzle of $\mathrm{Sr}_{2} \mathrm{RuO}_{4}$. Npj Quantum Mater. 2, 40 (2017). doi:https://doi. org/10.1038/s41535-017-0045-4

6. Maeno, Y., Hashimoto, H., Yoshida, K., Nishizaki, S., Fujita, T., Bednorz, J., Lichtenberg, F.: Superconductivity in a layered perovskite without copper. Nature. 372, 532-534 (1994). https://doi.org/ $10.1038 / 372532 \mathrm{a} 0$

7. Mackenzie, A.P., Julian, S.R., Diver, A.J., McMullan, G.J., Ray, M.P., Lonzarich, G.G., Maeno, Y., Nishizaki, S., Fujita, T.: Quantum oscillations in the layered perovskite superconductor $\mathrm{Sr}_{2} \mathrm{RuO}_{4}$. Phys. Rev. Lett. 76, 3786-3789 (1996). https://doi.org/ 10.1103/PhysRevLett.76.3786

8. Bergemann, C., Julian, S.R., Mackenzie, A.P., NishiZaki, S., Maeno, Y.: Detailed topography of the Fermi surface of $\mathrm{Sr}_{2} \mathrm{RuO}_{4}$. 
Phys. Rev. Lett. 84, 2662-2665 (2000). https://doi.org/10.1103/ PhysRevLett.84.2662

9. Bergemann, C., Mackenzie, A.P., Julian, S.R., Forsythe, D., Ohmichi, E.: Quasi-two-dimensional Fermi liquid properties of the unconventional superconductor $\mathrm{Sr}_{2} \mathrm{RuO}_{4}$. Adv. Phys. 52, 639-725 (2003). https://doi.org/10.1080/00018730310001621737

10. Rice, T., Sigrist, M.: $\mathrm{Sr}_{2} \mathrm{RuO}_{4}$ - an Electronic Analog of He-3. J. Phys.-Condens. Matter. 7, L643-L648 (1995). https://doi.org/10. 1088/0953-8984/7/47/002

11. Mackenzie, A.P., Haselwimmer, R.K.W., Tyler, A.W., Lonzarich, G.G., Mori, Y., Nishizaki, S., Maeno, Y.: Extremely strong dependence of superconductivity on disorder in $\mathrm{Sr}_{2} \mathrm{RuO}_{4}$. Phys. Rev. Lett. 80, 161-164 (1998). https://doi.org/10.1103/PhysRevLett.80.161

12. Mao, Z.Q., Mori, Y., Maeno, Y.: Suppression of superconductivity in $\mathrm{Sr}_{2} \mathrm{RuO}_{4}$ caused by defects. Phys. Rev. B. 60, 610 (1999)

13. Kikugawa, N., Maeno, Y.: Non-Fermi-liquid behavior in $\mathrm{Sr}_{2} \mathrm{RuO}_{4}$ with nonmagnetic impurities. Phys. Rev. Lett. 89, (2002). https:// doi.org/10.1103/PhysRevLett.89.117001

14. Kikugawa, N., Mackenzie, A.P., Bergemann, C., Borzi, R.A., Grigera, S.A., Maeno, Y.: Rigid-band shift of the Fermi level in the strongly correlated metal: $\mathrm{Sr}_{2-y} \mathrm{La}_{\mathrm{y}} \mathrm{RuO}_{4}$. Phys. Rev. B. 70, (2004). https://doi.org/10.1103/PhysRevB.70.060508

15. Mao, Z.Q., Maeno, Y., Fukazawa, H.: Crystal growth of $\mathrm{Sr}_{2} \mathrm{RuO}_{4}$. Mater. Res. Bull. 35, 1813-1824 (2000). https://doi.org/10.1016/ S0025-5408(00)00378-0

16. Bobowski, J.S., Kikugawa, N., Miyoshi, T., Suwa, H., Xu, H., Yonezawa, S., Sokolov, D.A., Mackenzie, A.P., Maeno, Y.: Improved single-crystal growth of $\mathrm{Sr}_{2} \mathrm{RuO}_{4}$. Condens. Matter. 4, 6 (2019). https://doi.org/10.3390/condmat4010006

17. Luke, G.M., Fudamoto, Y., Kojima, K.M., Larkin, M.I., Merrin, J., Nachumi, B., Uemura, Y.J., Maeno, Y., Mao, Z.Q., Mori, Y., Nakamura, H., Sigrist, M.: Time-reversal symmetry breaking superconductivity in $\mathrm{Sr}_{2} \mathrm{RuO}_{4}$. Nature. 394, 558-561 (1998). https:// doi.org/10.1038/29038

18. Ishida, K., Mukuda, H., Kitaoka, Y., Asayama, K., Mao, Z.Q., Mori, Y., Maeno, Y.: Spin-triplet superconductivity in $\mathrm{Sr}_{2} \mathrm{RuO}_{4}$ identified by O-17 Knight shift. Nature. 396, 658-660 (1998). https://doi.org/10.1038/25315

19. Ishida, K., Mukuda, H., Kitaoka, Y., Mao, Z.Q., Fukazawa, H., Maeno, Y.: $\mathrm{Ru}$ NMR probe of spin susceptibility in the superconducting state of $\mathrm{Sr}_{2} \mathrm{RuO}_{4}$. Phys. Rev. B. 63, 60507 (2001). https://doi.org/10.1103/PhysRevB.63.060507

20. Xia, J., Maeno, Y., Beyersdorf, P.T., Fejer, M.M., Kapitulnik, A.: High resolution polar Kerr effect measurements of $\mathrm{Sr}_{2} \mathrm{RuO}_{4}$ : Evidence for broken time-reversal symmetry in the superconducting state. Phys. Rev. Lett. 97, 167002 (2006). https:// doi.org/10.1103/PhysRevLett.97.167002

21. Kidwingira, F., Strand, J.D., Van Harlingen, D.J., Maeno, Y.: Dynamical superconducting order parameter domains in $\mathrm{Sr}_{2} \mathrm{RuO}_{4}$. Science. 314, 1267-1271 (2006). https://doi.org/10. 1126/science. 1133239

22. Nelson, K.D., Mao, Z.Q., Maeno, Y., Liu, Y.: Odd-parity superconductivity in $\mathrm{Sr}_{2} \mathrm{RuO}_{4}$. Science. 306, 1151-1154 (2004). https://doi. org/10.1126/science. 1103881

23. Ivanov, D.A.: Non-abelian statistics of half-quantum vortices in $\mathrm{p}$ wave superconductors. Phys. Rev. Lett. 86, 268-271 (2001). https://doi.org/10.1103/PhysRevLett.86.268

24. Lupien, C., MacFarlane, W.A., Proust, C., Taillefer, L., Mao, Z.Q., Maeno, Y.: Ultrasound attenuation in $\mathrm{Sr}_{2} \mathrm{RuO}_{4}$ : an angle-resolved study of the superconducting gap function. Phys. Rev. Lett. 86, 5986-5989 (2001). https://doi.org/10.1103/PhysRevLett.86.5986

25. Kirtley, J.R., Kallin, C., Hicks, C.W., Kim, E.-A., Liu, Y., Moler, K.A., Maeno, Y., Nelson, K.D.: Upper limit on spontaneous supercurrents in $\mathrm{Sr}_{2} \mathrm{RuO}_{4}$. Phys. Rev. B. 76, 14526 (2007). https://doi. org/10.1103/PhysRevB.76.014526

26. Hicks, C.W., Kirtley, J.R., Lippman, T.M., Koshnick, N.C., Huber, M.E., Maeno, Y., Yuhasz, W.M., Maple, M.B., Moler, K.A.: Limits on superconductivity-related magnetization in $\mathrm{Sr}_{2} \mathrm{RuO}_{4}$ and $\mathrm{PrO}_{4} \mathrm{Sb}_{12}$ from scanning SQUID microscopy. Phys. Rev. B. 81, 214501 (2010). https://doi.org/10.1103/PhysRevB.81.214501

27. Huang, W., Lederer, S., Taylor, E., Kallin, C.: Nontopological nature of the edge current in a chiral p-wave superconductor. Phys. Rev. B. 91, 94507 (2015). https://doi.org/10.1103/PhysRevB.91. 094507

28. Scaffidi, T., Simon, S.H.: Large Chern number and edge currents in $\mathrm{Sr}_{2} \mathrm{RuO}_{4}$. Phys. Rev. Lett. 115, 87003 (2015). https://doi.org/10. 1103/PhysRevLett.115.087003

29. Yonezawa, S., Kajikawa, T., Maeno, Y.: First-order superconducting transition of $\mathrm{Sr}_{2} \mathrm{RuO}_{4}$. Phys. Rev. Lett. 110, 77003 (2013). https://doi.org/10.1103/PhysRevLett.110.077003

30. Yonezawa, S., Kajikawa, T., Maeno, Y.: Specific-heat evidence of the first-order superconducting tTransition in $\mathrm{Sr}_{2} \mathrm{RuO}_{4}$. J. Phys. Soc. Jpn. 83, 83706 (2014). https://doi.org/10.7566/JPSJ.83. 083706

31. Hicks, C.W., Barber, M.E., Edkins, S.D., Brodsky, D.O., Mackenzie, A.P.: Piezoelectric-based apparatus for strain tuning. Rev. Sci. Instrum. 85, 65003 (2014). https://doi.org/10.1063/1. 4881611

32. Hicks, C.W., Brodsky, D.O., Yelland, E.A., Gibbs, A.S., Bruin, J.A.N., Barber, M.E., Edkins, S.D., Nishimura, K., Yonezawa, S., Maeno, Y., Mackenzie, A.P.: Strong increase of $\mathrm{T}_{\mathrm{c}}$ of $\mathrm{Sr}_{2} \mathrm{RuO}_{4}$ under both tensile and compressive strain. Science. 344, 283-285 (2014). https://doi.org/10.1126/science.1248292

33. Watson, C.A., Gibbs, A.S., Mackenzie, A.P., Hicks, C.W., Moler, K.A.: Micron-scale measurements of low anisotropic strain response of local $\mathrm{T}_{\mathrm{c}}$ in $\mathrm{Sr}_{2} \mathrm{RuO}_{4}$. Phys. Rev. B. 98, 094521 (2018). https://doi.org/10.1103/PhysRevB.98.094521

34. Steppke, A., Zhao, L., Barber, M.E., Scaffidi, T., Jerzembeck, F., Rosner, H., Gibbs, A.S., Maeno, Y., Simon, S.H., Mackenzie, A.P., Hicks, C.W.: Strong peak in $\mathrm{T}_{\mathrm{c}}$ of $\mathrm{Sr}_{2} \mathrm{RuO}_{4}$ under uniaxial pressure. Science. 355, eaaf9398 (2017)

35. Barber, M.E., Gibbs, A.S., Maeno, Y., Mackenzie, A.P., Hicks, C.W.: Resistivity in the Vicinity of a van Hove singularity: $\mathrm{Sr}_{2} \mathrm{RuO}_{4}$ under uniaxial pressure. Phys. Rev. Lett. 120, 076602 (2018). doi:https://doi.org/10.1103/PhysRevLett.120.076602

36. Raghu, S., Bauer, E.D., Brown, S.E., Constraints on the superconducting order parameter in $\mathrm{Sr}_{2} \mathrm{RuO}_{4}$ from oxygen-17 nuclear magnetic resonance. Nature 574, 74 (2019)

37. Ishida, K., Manago, M., Maeno, Y., Reduction of the ${ }^{17} \mathrm{O}$ Knight shift in the superconducting state and the heat-up effect by NMR pulses on $\mathrm{Sr}_{2} \mathrm{RuO}_{4}$. ArXiv Prepr. ArXiv190712236. (2019)

38. Scaffidi, T., Romers, J.C., Simon, S.H.: Pairing symmetry and dominant band in $\mathrm{Sr}_{2} \mathrm{RuO}_{4}$. Phys. Rev. B. 89, 220510 (2014). https:// doi.org/10.1103/PhysRevB.89.220510

Publisher's Note Springer Nature remains neutral with regard to jurisdictional claims in published maps and institutional affiliations. 